\title{
PALEOAMBIENTE DEPOSICIONAL E ORIGEM DA MATÉRIA ORGÂNICA DOS FOLHELHOS BETUMINOSOS DA FORMAÇÃO IPUBI, PORÇÕES LESTE E SUDESTE DA BACIA DO ARARIPE, NE DO BRASIL: EVIDÊNCIA DE $N$-ALCANOS E ISOPRENOIDES
}

\subsection{0/1980-8208/estudosgeologicos.v30n1p31-44}

Willian Alexandre Lima de Moura ${ }^{1}$ João Carlos Morais de Araújo ${ }^{1}$ Thales Lúcio ${ }^{1} \&$ João Adauto de Souza Neto ${ }^{1,2}$

${ }^{1}$ Programa de Pós Graduação em Geociências-PPGEOC, Universidade Federal de Pernambuco-UFPE, Av. da Arquitetura, s/n, CEP 507740-550. E-mail: willian.moura@ufpe.br; jc.morais.araujo@gmail.com; thales.lucio@ufpe.br ${ }^{2}$ Departamento de Geologia, Universidade Federal de Pernambuco-UFPE, Av. da Arquitetura, s/n, CEP 507740-550.E-mail: adauto@ufpe.br; joao.souzant@ufpe.br

\section{RESUMO}

Condições paleoambientais dos folhelhos betuminosos cretáceos da Formação Ipubi (Bacia do Araripe-NE do Brasil) são continuamente debatidos. O principal ponto de controvérsia é se essas rochas foram depositadas em condições lacustres ou marinhas restritas. O presente estudo se desenvolveu com amostras representativas desses folhelhos betuminosos da unidade sedimentar supracitada, a partir da análise de biomarcadores saturados ( $n$-alcanos e isoprenoides) para se reconstruir o paleoambiente deposicional e a origem da matéria orgânica. O predomínio de $n$-alcanos entre $n-\mathrm{C}_{13}$ e $n$ - $\mathrm{C}_{19}$ são indicativos de uma contribuição algálica. Entretanto, picos das concentrações dos $n$ alcanos $n-\mathrm{C}_{27}, n-\mathrm{C}_{28}$ e $n-\mathrm{C}_{29}$ sugerem uma influência de plantas continentais. As relações $\mathrm{Pr} / \mathrm{Fi}$ e $\mathrm{Pr} / \mathrm{Fi}$ vs. $\left(\mathrm{Pr}+n-\mathrm{C}_{17}\right) /\left(\mathrm{Fi}+n-\mathrm{C}_{18}\right)$ indicam condições redutoras (baixa oxigenação) e hipersalinas durante a deposição destes folhelhos. O diagrama $\mathrm{Pr} / n-\mathrm{C}_{17} v s$. Fi $/ n-\mathrm{C}_{18}$, além de sugerir também condições redutoras durante a deposição destes folhelhos, classifica a contribuição algálica como de origem marinha-transicional. Sendo assim, baseado nos compostos orgânicos moleculares, este estudo sugere que os folhelhos estudados foram depositados em condições anóxicas e hipersalinas em um paleoambiente marinho restrito à transicional.

Palavras chave: Folhelhos betuminosos, Biomarcadores, Formação Ipubi, Bacia do Araripe

\begin{abstract}
Paleoenvironmental conditions from cretaceous black shales of Ipubi Formation (Araripe Basin-NE Brasil) are continuously debated. The main point of controversy is if these rocks were deposited in a lacustrine or restricted marine environment. The present study was developed in these black shales, by saturated biomarkers analyses ( $n$-alkanes and isoprenoids) to discuss about the paleoenvironment of deposition of those shales. The $n$ alkanes predominance between $n-\mathrm{C}_{13}$ and $n-\mathrm{C}_{19}$ reveal algalic contribution. However an influence of terrestrial organism is also observed by the presence of $n-\mathrm{C}_{27}, n-\mathrm{C}_{28}$, and $n$ $\mathrm{C}_{29}$. The $\mathrm{Pr} / \mathrm{Ph}$ ratio and $\mathrm{Pr} / \mathrm{Ph} v s$. $\left(\mathrm{Pr}+n-\mathrm{C}_{17}\right) /\left(\mathrm{Fi}+n-\mathrm{C}_{18}\right)$ diagram indicated both anoxic and hypersaline conditions during the deposition of those shales. The $\mathrm{Pr} / n-\mathrm{C}_{17} v s . \mathrm{Ph} / n$ -
\end{abstract}


$\mathrm{C}_{18}$ diagram also suggests reducing conditions, and classify an algalic contribution as marine-transitional source. Thus, this study indicates that the investigated shales were deposited in reducing and hypersaline conditions in a restricted marine to transitional environment.

Keywords: Black shale; Biomarkers; Ipubi Formation; Araripe Basin

\section{INTRODUÇÃO}

Biomarcadores constituem fósseis moleculares formados a partir de antigos organismos viventes tais como bactérias, algas, plantas superiores, e animais invertebrados (Tissot \&Welte, 1984; Killops \& Killops, 2004; Peters et al., 2005). A utilização dos biomarcadores é possível devido à preservação de estruturas moleculares primárias de seus precursores biológicos (Peters \& Moldowan, 1993; Peters et al., 2005; Killops \& Killops, 2005). Portanto, sua aplicação está na reconstrução paleoambiental, no grau de maturação e origem da matéria orgânica (M.O.) através de biomarcadores existentes em rochas sedimentares com acumulação de matéria orgânica.

$\mathrm{O}$ estudo de biomarcadores em bacias sedimentares do Nordeste (e.g. bacias do Reconcavo, Araripe, Souza, São Luís) foram anteriormente investigados por trabalhos como os de Penteado \& Behar, (2000); Carvalho et al. (2013); Casilli et al. (2014); Castro et al. (2017); Gonzalez et al. (2020). Especificamente na Bacia do Araripe, o estudo de Biomarcadores já foi preteritamente estudado por Castro et al. (2017), mas geograficamente restrito ao setor oeste e norte da bacia enfocada, sendo a contribuição deste manuscrito apresentar novos dados, em outras regiões da Bacia do Araripe, as das porções leste e sudoeste. Não obstante pretende fomentar uma contribuição acerca do debate sobre o ambiente deposicional da Formação Ipubi e sobre a possível contribuição marinha durante a deposição desta unidade estratigráfica
(Arai, 2012; Bobco et al., 2017; Goldberg et al., 2019; Lúcio et al., 2020).

Portanto o presente estudo contribui por meio da caracterização de biomarcadores saturados, através da distribuição de compostos $n$-alcanos e isoprenoides nos folhelhos betuminosos pertencentes à Formação Ipubi, Bacia do Araripe. Desse modo, pretende-se obter uma melhor compreensão do contexto paleoambiental em que estes folhelhos estariam relacionados, além da origem da matéria orgânica que perfaz essas rochas.

\section{CONTEXTO GEOLÓGICO}

A Bacia Sedimentar do Araripe está localizada no Nordeste brasileiro (Fig. 1). De acordo com Assine (2007), a origem desta bacia está relacionada com a fragmentação do continente Gondwana e consequente abertura do Atlântico Sul. Neumann (1999), Assine (2007) e Neumann \& Assine (2015) revisaram e propuseram a estratigrafia desta bacia sedimentar, onde estão descritas quatro tectonosequências, separadas por discordâncias regionais, e indicadas na Figura 2.

I) Sequência Beta: marcada pela Formação Cariri composta por arenitos com níveis conglomeráticos depositados em sistema fluvial entrelaçado (Silvestre et al., 2017);

II) Sequência Pré-rifte representada pelas formações Missão Velha e Brejo Santo, composta por arenitos, argilitos vermelhos e 
verdes depositados em sistema lacustre (Fambrini et al., 2012);

III) Sequência Sin-rifte caracterizada pela Formação Abaiara, composta por intercalação de arenitos, argilitos, folhelhos e conglomerados (Fambrini et al., 2019) depositados em ambiente fluvio-deltaico-lacustre (Scherer $e t$ al., 2014);

IV) Sequência Pós-rifte representada pelos grupos: Santana (formações Barbalha, Crato, Ipubi e Romualdo) e Araripe (formações Exu e Araripina). O grupo Santana inicia com a deposição da Formação Barbalha que corresponde à deposição de arenitos, argilitos vermelhos e amarelados e folhelhos relacionados a um ambiente flúvio-lacustre (Fambrini et al., 2015; Fambrini et al., 2019); calcários laminados e folhelhos da Formação Crato depositados em sistema lacustre (Neumann, 1999); folhelhos, argilitos, margas e evaporitos na Formação Ipubi (Lúcio et al., 2016); arenitos, calcários, folhelhos na Formação Romualdo depositados em ambiente marinho raso de (Assine et al., 2014) de idade Aptiana inferior (biozona P-270; Arai \& Assine, 2020). O grupo Araripe está relacionado à deposição de arenitos e argilitos em ambiente lacustre e fluvial das formações Exu e Araripina (Assine, 2007) 


\section{PALEOAMBIENTE DEPOSICIONAL E ORIGEM DA MATÉRIA...}

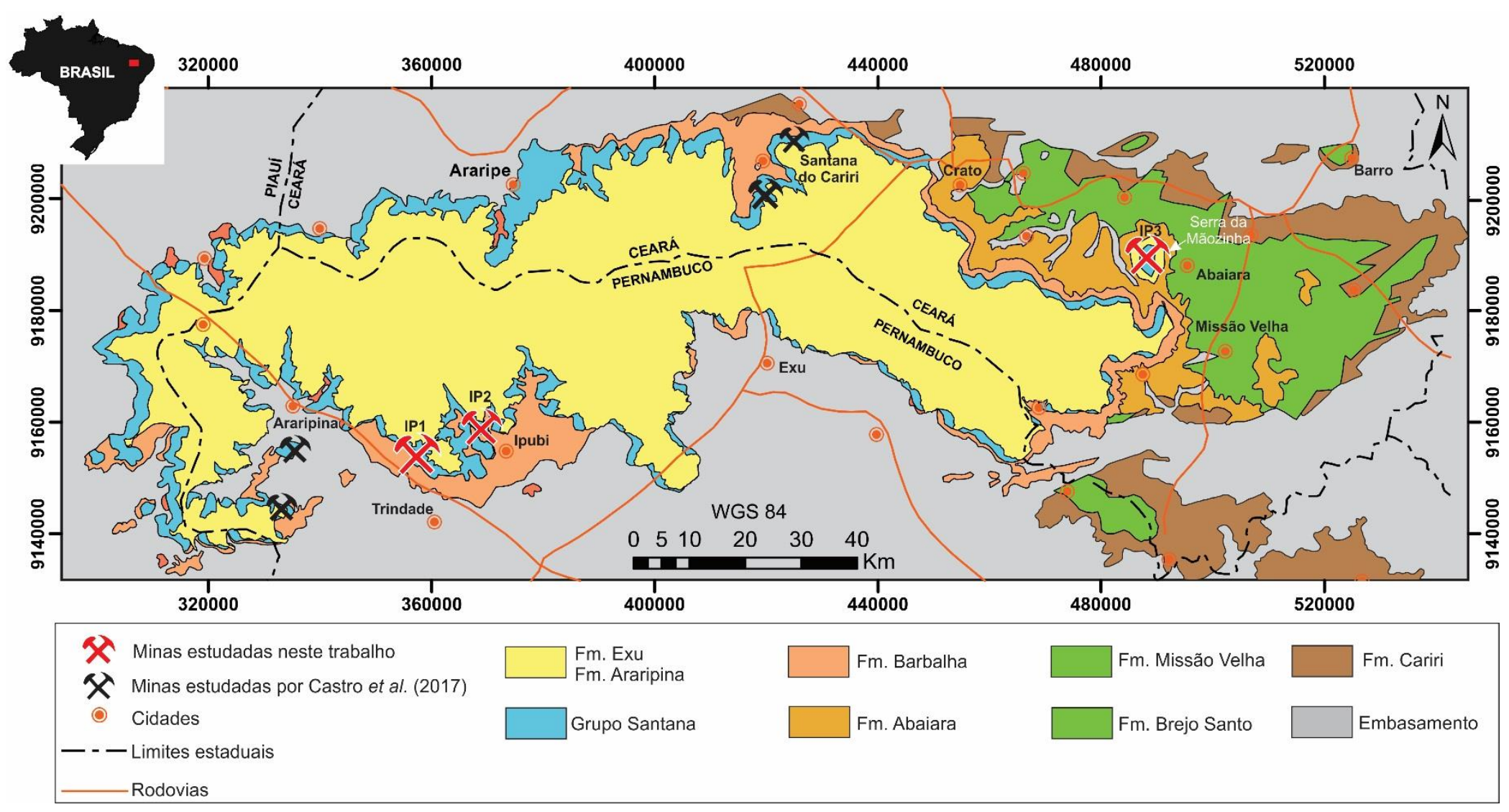

Figura 1: Mapa geológico simplificado da Bacia do Araripe. Destaca-se a localização das minas de gipsita, onde foram coletadas as amostras de folhelhos betuminosos da Formação Ipubi. Modificado de Assine (2007), Fabin et al. (2018). 


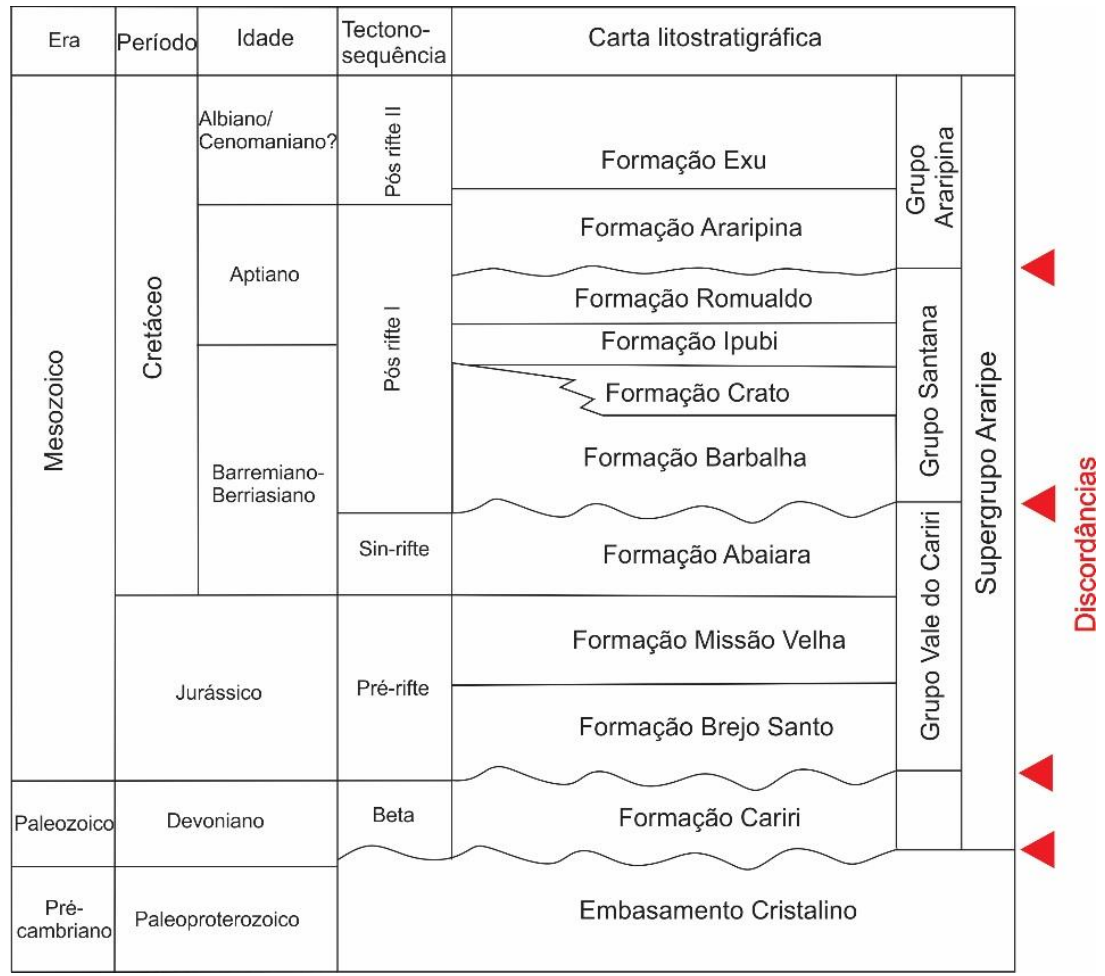

Figura 2: Diagrama estratigráfico da Bacia do Araripe. Adaptado de Neumann (1999); Neumann \& Assine (2015); seguindo novos intervalos cronoestratigráficos obtidos por Lúcio et al. (2020) na Formação Ipubi e por Arai \& Assine, (2020) na Formação Romualdo.

\section{Formação Ipubi}

A Formação Ipubi definida por Neumann (1999), Assine et al. (2014) e Neumann \& Assine (2015) é caracterizada pela ocorrência de cerca de 20-25 m de evaporitos (gipsita e anidrita subordinada), sobrepostos a uma camada de 5-7 $\mathrm{m}$ de folhelhos betuminosos (Neumann, 1999). Neumann (1999) caracterizou esta formação sedimentar como uma evolução do paleolago Araripe além de elevar ao status de Formação e não mais de "Camada Ipubi" ou "Membro Ipubi", sendo esta classificação proposta por Neumann (1999) aqui seguida. Arai \& Coimbra (1990) e Coimbra et al. (2002) caracterizaram a assembleia fossilífera existente nesta formação contendo dinoflagelados, ostracodes não marinhos, grãos de pólen, foraminíferos e moluscos, indicando a presença de influência marinha nesta unidade estratigráfica. Assine (2007) através de descrição faciológica propõe para a formação Ipubi um ambiente de Sabhkas Costeiros sem um contato direto com o mar. Nascimento Jr. et al. (2016) com base em descrição faciológica argumenta um sistema deposicional tipo Playa Lake sem nenhuma conexão direta com o mar. Arai (2012), Arai (2014), Goldberg et al. (2019) com base na descrição de palinoforaminíferos sub-ordem Rotalina defenderam uma ingressão marinha tetiana nos folhelhos da Formação Ipubi, preteritamente à Formação Romualdo (unidade a qual se associa a primeira ingressão marinha cretácea da Bacia do Araripe). Castro et al. (2017), com base em biomarcadores saturados e 
aromáticos, retoma a ideia de um sistema deposicional lacustre salino para os folhelhos da Formação Ipubi. Lúcio et al. (2020), com base em dados isotópicos de Re/Os, propõem paleoambiente marinho restrito, e estabelece um marco geocronológico absoluto nesta bacia através de uma idade de $123 \pm 3,5 \mathrm{Ma}$ (Barremiano Superior/Aptiano Inferior).

Em relação aos estudos sobre geoquímica orgânica nos folhelhos betuminosos da Formação Ipubi, destaca-se o trabalho de Delgado (2012) que estudou uma amostra da região norte (Mina Pedra Branca) da bacia e apresentou dados de Pirólise, Hidropirólise, Carbono orgânico Total (COT), $n$-alcanos e isoprenoides. Castro et al. (2017) realizaram análises de biomarcadores ( $n$-alcanos, isoprenóides, terpanos e esteranos além de compostos aromáticos) em quatro amostras de folhelhos betuminosos da Formação Ipubi. Castro et al. (2017) indicaram condições de deposição em ambiente anóxico, matéria orgânica lacustre e uma acumulação de matéria orgânica entre 20-30\%, indicando estes folhelhos como potenciais rochas geradoras para a Bacia do Araripe.

\section{MATERIAL E MÉTODOS}

Três amostras regionalmente representativas foram selecionadas para análises geoquímicas orgânicas (biomarcadores saturados). A localização das amostras está identificada na Figura 1. A extração do betume foi realizada no Laboratório de Estudos Orgânicos Marinhos (ORGANOMAR-UFPE) por meio de Extrator Sohxlet ${ }^{\circledR}$ com uso de $30 \mathrm{ml}$ diclorometanol por $24 \mathrm{~h}$. Em seguida, realizou-se a cromatografia líquida em coluna de sílica no Laboratório de Geoquímica Aplicada ao Petróleo (LGAP-UFPE). Foram utilizados $10 \mathrm{ml}$ de $n$-hexano, para extração dos compostos saturados; uma solução de 8 $\mathrm{ml}$ de $n$-hexano e $2 \mathrm{ml}$ de diclorometano, para extração dos compostos saturados; uma solução de $9 \mathrm{ml}$ de diclorometano e $1 \mathrm{ml}$ de metanol, para extração dos compostos polares (NSO).

A análise por cromatografia gasosa acoplada a espectrômetro de massas (CG-MS) foi realizada no Laboratório de Saneamento Ambiental (LSA-UFPE). Foi utilizado um cromatógrafo modelo Agilent 7890A. As análises foram realizadas por modo de varredura linear (SCAN). As condições cromatográficas foram estabelecidas da seguinte forma: $70{ }^{\circ} \mathrm{C}$ de temperatura inicial com taxa de aquecimento de $20{ }^{\circ} \mathrm{C} / \mathrm{min}$, até a temperatura de $170{ }^{\circ} \mathrm{C}$, seguindo uma taxa de aquecimento de $2{ }^{\circ} \mathrm{C} / \mathrm{min}$ até a temperatura final de $310^{\circ} \mathrm{C}$ mantendo-se a isoterma por $5 \mathrm{~min}$. A temperatura do injetor foi de $290{ }^{\circ} \mathrm{C}$ e do detector a 310 ${ }^{\circ} \mathrm{C}$. A injeção ocorreu sem divisão de fluxo e hélio como gás carreador. A interpretação dos dados se deu através da extração seletiva de íons utilizando a relação massa/carga $(\mathrm{m} / \mathrm{z})$. Foi utilizada a relação $\mathrm{m} / \mathrm{z}=85$ referente aos $n$ alcanos e isoprenoides.

\section{RESULTADOS Caracterização Geológica}

Os folhelhos betuminosos da Formação Ipubi ocorrem subjacentes à camada de evaporitos da mesma unidade estratigráfica e estas duas litologias encontram-se estratigraficamente posicionadas abaixo da Formação Romualdo (Fig. 3A). Os afloramentos representativos destes folhelhos são expostos nas cavas das minas de explotação de gipsita existentes no Pólo Gesseiro de Pernambuco (Fig. 3A). Os afloramentos de folhelhos são compostos por uma exposição de aproximadamente $30-50 \mathrm{~cm}$ de espessura, iniciando na base por um nível de argilito cinza a preto que passa gradualmente para um nível margoso de coloração cinza escuro, seguido no topo 
por folhelhos betuminosos sotopostos pela camada de gipsita (Figs. 3B, 3C). O contato com a Formação Romualdo é marcado por uma superfície discordante (Fig.3A). Os folhelhos betuminosos apresentam fissilidade como estrutura sedimentar principal. Ocorrem nestes folhelhos intercalações de veios de gipsita (aproximadamente $5-10 \mathrm{~cm}$ de espessura) em algumas exposições (Fig. 3B).
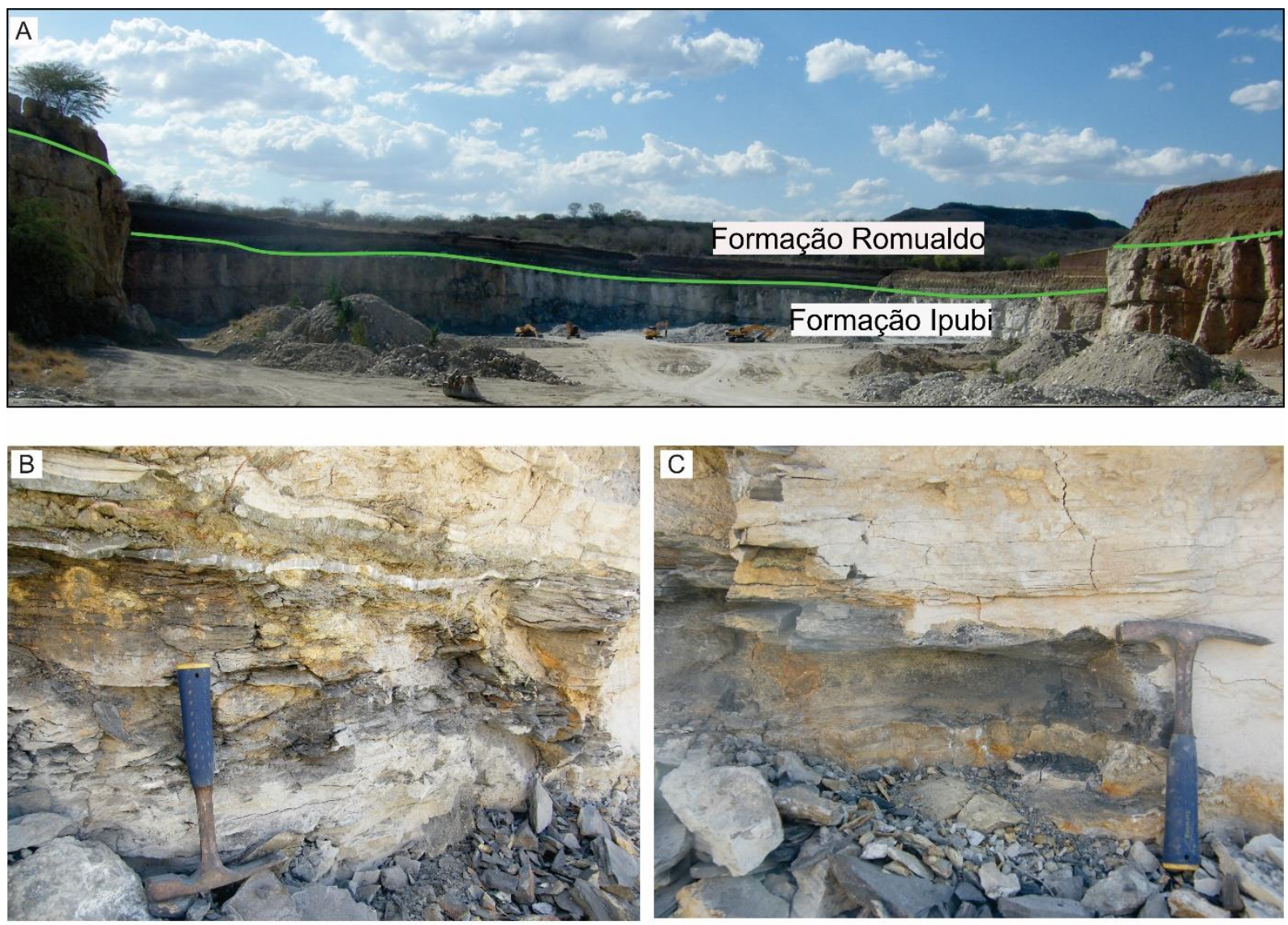

Figura 3: Aspectos gerais dos afloramentos da Formação Ipubi. (A) Mina de Gipsita MPGesso S.A, destacando o contato entre a Formação Ipubi e a Formação Romualdo. (B, C) Exposição de folhelho betuminoso da Formação Ipubi, subjacente à camada de gipsita.

\section{Análises de Geoquímica Orgânica}

Os cromatogramas de íons totais das três amostras analisadas são apresentados na Figura 4. De modo geral, as amostras analisadas apresentaram poucos sinais de biodegradação/oxidação da matéria orgânica. Apenas a amostra IP2MPGesso apresentou biodegradação da matéria orgânica, indicada pelo aumento do nível de base do cromatograma (baseando-se nos critérios interpretativos propostos por Gonzalez et al., 2020; Fig. 4A). 


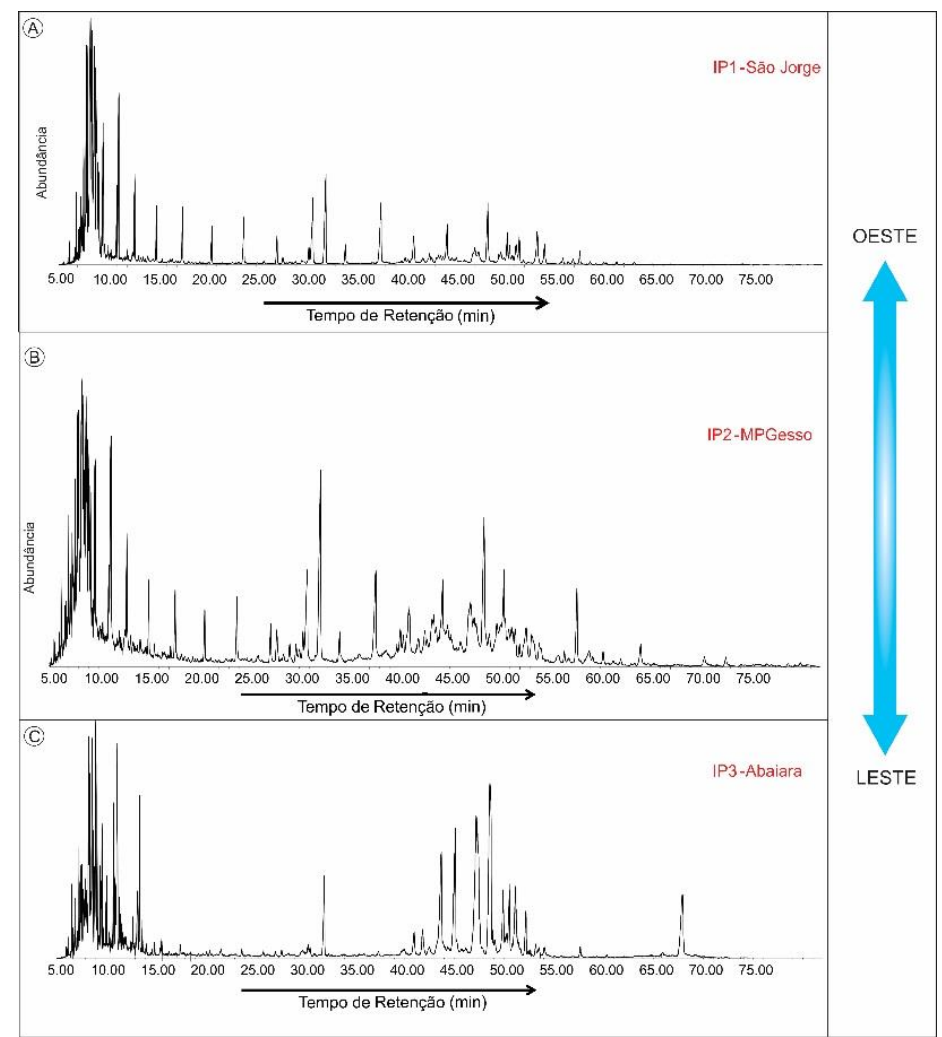

Figura 4. Cromatograma de íons totais, da fração saturada analisada, extraído do betume dos folhelhos betuminosos da Formação Ipubi. A) Cromatograma de íons totais da amostra da mina MPGesso; B) Cromatograma de íons totais da amostra da mina São Jorge; C) Cromatograma de íons totais da amostra da mina da localidade de Mudubim, próxima à cidade de Abaiara.

O cromatograma de íons extraídos $(m / z$ 85) correspondente aos $n$-alcanos e isoprenoides mostra um predomínio de abundância de carbonos do $n-\mathrm{C}_{14}$ ao $n$ $\mathrm{C}_{19}$, Entretanto, são identificados a presença de carbonos superiores ao $n-\mathrm{C}_{19}$ (Figura 5). Apenas a amostra IP3Abaiara apresentou baixa concentração nos carbonos superiores a $n$-C19. Não obstante, são identificados os isoprenoides: pristano e fitano logo após a identificação do carbono $n-\mathrm{C}_{17}$ e $n-\mathrm{C}_{18}$ (Fig. 5). Os valores da razão Pristano/Fitano $(\mathrm{Pr} / \mathrm{Fi})$ variaram entre
0,21 a 0,24 . O diagrama $\mathrm{Pr} / \mathrm{Fi}$ vs. $(\mathrm{Pr}+n-$ $\left.\mathrm{C}_{17}\right) /\left(\mathrm{Fi}+n-\mathrm{C}_{18}\right)$, seguindo-se a proposta de Peters et al. (2005), também indicou condições redutoras para as amostras analisadas (Figura 6A). O diagrama $\mathrm{Pr} / n$ $\mathrm{C}_{17}$ vs. $\mathrm{Fi} / n-\mathrm{C}_{18}$ (proposto por Peters et al., 2005) indicou um predomínio de algas marinhas em condições redutoras, como fonte principal de matéria orgânica e Eh do paleoambiente de deposição dos folhelhos betuminosos investigados (Fig. 6B). Todos estes valores encontram-se reportados na Tabela 1. 
Tabela 1: Valores obtidos para os principais parâmetros geoquímicos a partir da análise de compostos orgânicos saturados em amostras de folhelhos betuminosos da Formação Ipubi. Os valores foram obtidos a partir do cálculo da área do pico no cromatograma.

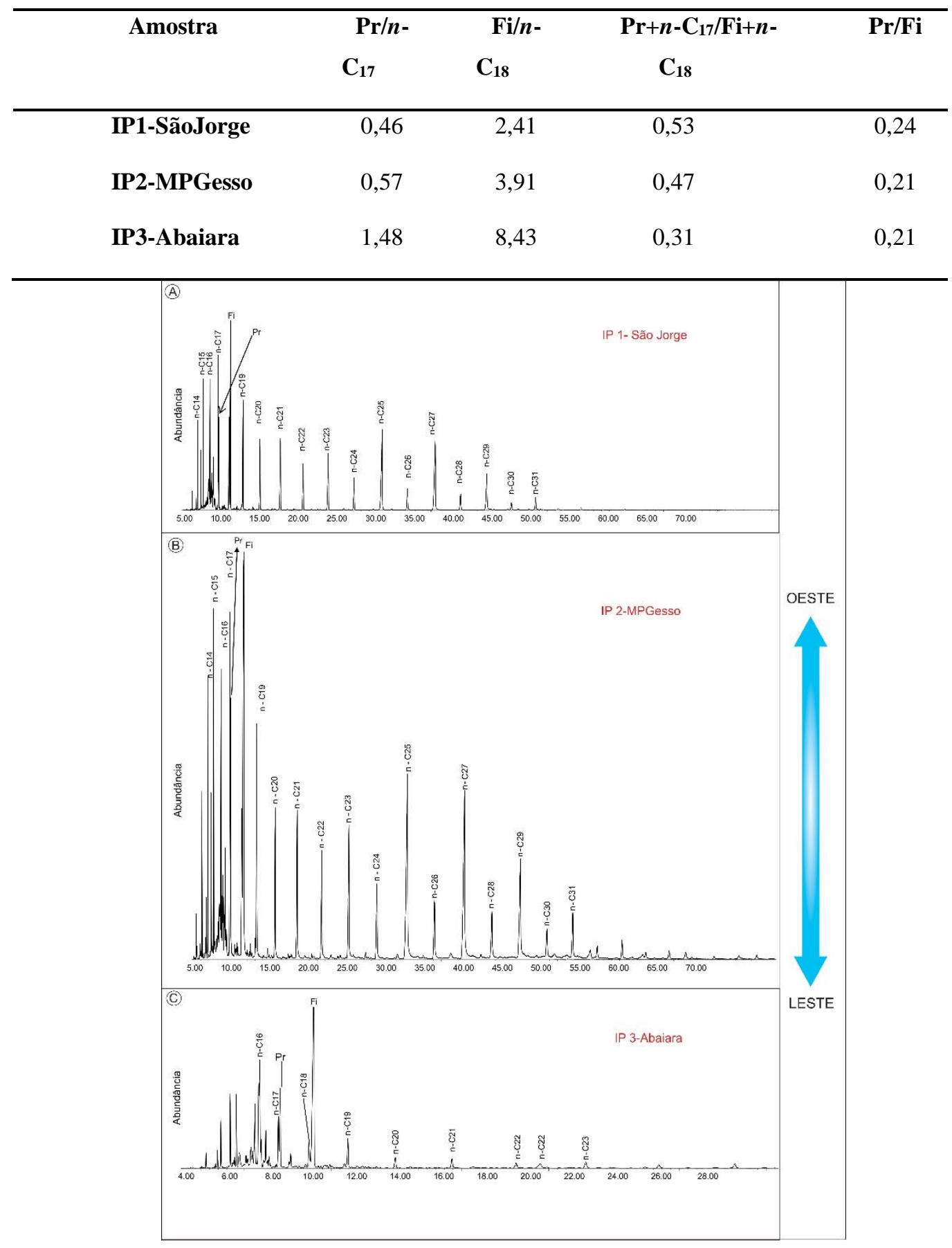

Figura 5. Cromatograma de íons extraídos (m/z 85) série dos n-alcanos e isoprenóides, para os folhelhos betuminosos da Formação Ipubi. A) Amostra referente à mina São Jorge; B) Amostra da mina MPGesso; C) amostra da localidade de Mudubim, próximo à cidade de Abaiara. 

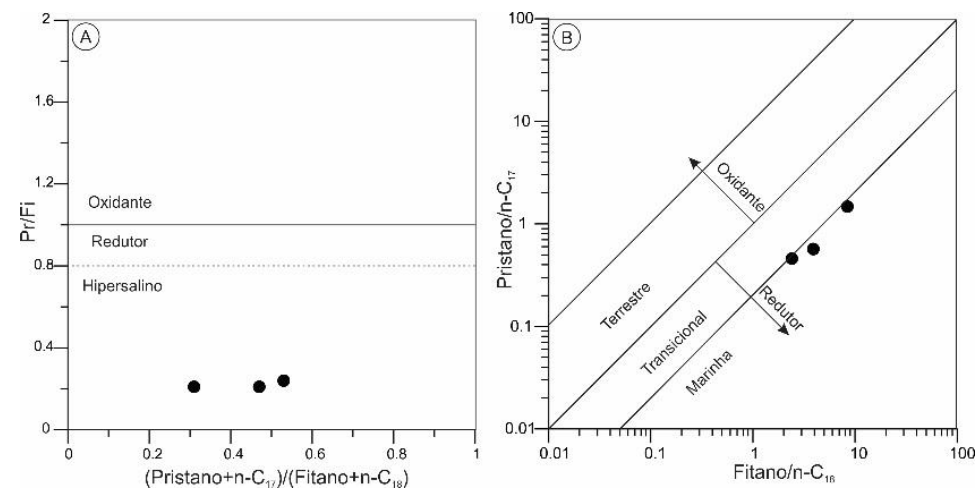

Figura 6. A) Diagrama $\mathrm{Pr} / \mathrm{Fi}$ vs. $\left(\mathrm{Pr}+n-\mathrm{C}_{17}\right) /\left(\mathrm{Fi}+n-\mathrm{C}_{18}\right)$ (proposto por Peters et al., 2005) indicando condições redutoras e hipersalinas para a deposição dos folhelhos betuminosos da Formação Ipubi. B) Diagrama Pristano/n- $\mathrm{C}_{17} v s$. Fitano/n- $\mathrm{C}_{18}$ (proposto por Peters et $a l$., 2005) indicando condições redutoras e uma contribuição de algas marinhastransicionais como fonte da matéria orgânica.

\section{DISCUSSÃO}

A identificação e importância de $n$ alcanos em amostras de betume representam a natureza de compostos lipídicos em precursores biológicos antigos (Killops \& Killops, 2005; Peters et al., 2005). A distribuição dos picos de $n$-alcanos são fatores confiáveis sobre a origem e tipo de matéria orgânica (Xia et al., 2019). De modo geral, organismos marinhos tendem a possuir um predomínio dos picos menores que $n$ $\mathrm{C}_{21}$, enquanto organismos terrestres possuem predomínio nos carbonos maiores que $n-\mathrm{C}_{27}$ (Hockun et al., 2016). Os dados aqui reportados sugerem que os precursores biológicos da matéria orgânica presente nos folhelhos betuminosos da Formação Ipubi foram algas e bactérias de origem marinha (Fig. 6B), depositadas em ambiente predominantemente redutor (Fig. 6A, 6B), para as amostras estudadas neste trabalho (minas São Jorge, MPGesso e Mudubim). Entretanto, uma contribuição de plantas superiores (origem terrestre) também é indicada pela presença dos picos de $n$-alcanos $\left(n-\mathrm{C}_{27}, n-\mathrm{C}_{28}\right.$ e $\left.n-\mathrm{C}_{29}\right)$, apenas no setor sudeste (este trabalho), e no setor oeste conforme indicado por Castro et al. (2017) sugerindo uma influência continental, o que indica uma mistura de fontes biológicas (Peters et al., 2005).

O paleoambiente deposicional da Formação Ipubi foi preteritamente discutido por alguns autores (e.g. Neumann, 1999; Assine, 2007; Assine et al., 2014; Arai, 2014; Castro et al., 2017; Bobco et al., 2017; Fabin et al., 2018; Goldberg et al., 2019; Lúcio et al., 2020). Entretanto, ainda existem divergências a respeito da contribuição (ou não) de águas marinhas durante a deposição dos mesmos. Os argumentos estabelecidos são a presença de dinoflagelados e palinoforaminíferos, organismos exclusivamente marinhos, em amostras destes folhelhos (Arai, 2009; Arai, 2014; Goldberg et al., 2019), além de uma assinatura isotópica de enxofre $(+10,27$ e $+17,99 \%)$ e de oxigênio $(+7,72 \mathrm{e}+13,30 \%$ o de origem marinha (Bobco et al., 2017) e assinaturas de ${ }^{187} \mathrm{Os} /{ }^{188} \mathrm{Os}(1,97 \pm 0,02)$ também sugestivas de condições marinhas (Lúcio et al., 2020). Por outro lado, a presença de ostracodes não marinhos Theriosynoecum ? Sp. (Coimbra et al., 2002; Souza et al., 2017) e fragmentos continentais como âmbar (Pereira et al., 2020) corroboram com a interpretação de um ambiente continental para estes folhelhos. 
A influência de algas marinhastransicionais apontadas neste estudo e a presença de outros organismos exclusivamente de origem marinha (Arai, 2009; Arai, 2014; Goldberg et al., 2019) leva-se a considerar a deposição destes folhelhos em um ambiente transicional a um marinho restrito, onde existiria a influência desde organismos marinhos a organismos continentais. Tal hipótese corroborada com evidências de uma transgressão marinha durante $o$ Aptiano nas bacias sedimentares do Nordeste (Arai, 2014; Assine et al., 2014; Lúcio et al., 2020).

Em relação ao potencial de geração de hidrocarbonetos por parte destes folhelhos, ressalta-se que os valores obtidos de COT nestes folhelhos variam entre 20-30\% (Castro et al., 2017), sugerindo uma excelente acumulação de matéria orgânica (Peters et al., 2005). Apesar disto, Delgado (2012) apontou uma baixa evolução térmica sofrida por estas rochas, indicando baixa taxa de transformação do querogênio para betume. Posteriormente, Lúcio et al. (2016) também indicaram baixas condições térmicas apontadas por elevadas concentrações de compostos polares (NSO) em relação aos compostos saturados e aromáticos dos betumes extraídos destes folhelhos. Além disso, Castro et al. (2017) reporta coeluição de hopanos e hopenos indicando compostos insaturados devido à baixa evolução térmica. Não obstante, Souza Neto et al. (2013) associam elevadas concentrações de $\mathrm{BaO}(\sim 2 \%)$ e de metais-traço como indicativos de condições hipersalinas e redutoras durante a deposição destes folhelhos. Estas condições ambientais são consideradas como os mecanismos responsáveis pela elevada acumulação de matéria orgânica nestes folhelhos. Porém, devido à baixa evolução térmica considerando toda a Bacia do Araripe, há uma dificuldade para o potencial de exploração convencional na mesma. Sendo uma alternativa a exploração por parte de meios não convencionais, o que caracterizam tais folhelhos como shale gas ou oil shale (Souza Neto et al., 2013).

\section{CONCLUSÕES}

A partir dos dados obtidos neste estudo pode se propor que os folhelhos betuminosos da Formação Ipubi sofreram uma influência marinhatransicional na deposição dos mesmos, o que é observada a partir das relações geoquímicas estabelecidas por Pristano/Fitano, $n$-C17 e $n$-C18, além do perfil de distribuição dos $n$-alcanos. Estes últimos também indicam uma contribuição predominante de algas e bactérias. Além disso, uma contribuição de plantas superiores (continentais) é observada na porção oeste e sudoeste da Bacia do Araripe. Em contra partida, uma baixa contribuição destes organismos é observada na porção leste da Bacia do Araripe, possivelmente indicando condições distais. Desta forma, esses folhelhos betuminosos foram depositados sob condições anóxicas, hipersalinas, em ambiente transicional sob influência da transgressão marinha Aptiana, nas bacias sedimentares do Nordeste brasileiro.

\section{Agradecimentos}

Os autores agradecem ao $\mathrm{CNPq}$, CAPES e PRH-AnP pelo financiamento das bolsas de iniciação científica, mestrado e doutorado. J.A.S.N. agradece pela sua bolsa de produtividade (processo. $\mathrm{N}^{\circ}$ : 312.275/2017-0). As minas de gipsita São Jorge S.A. e MPGesso S.A., pelo acesso a coleta das amostras. Agradecemos a Prof. Dra. Eliete Zanardi-Lamardo pelo acesso ao Organomar e a equipe do LSA, na pessoa do Prof. Dr. Mário Kato, pelo uso do CG-MS. 


\section{REFERÊNCIAS}

Arai, M., 2014. Aptian/Albian (Early Cretaceous) paleogeography of the South Atlantic: a paleontological perspective. Brazilian Journal of Geology, 44: 339- 350.

Arai, M., 2012. Evidência micropaleontológica da ingressão marinha aptiana (pré-evaporítica) na Bacia do Araripe, Nordeste do Brasil. In: 46 Congresso Brasileiro de Geologia... Volume único p.1.

Arai, M., Coimbra, J. C., 1990. Análise paleoecológica do registro das primeiras ingressões marinhas na Formação Santana (Cretáceo Inferior da Chapada do Araripe). Simpósio Bacia do Araripe e Bacias Interiores do Nordeste, 15p. Assine, M. L., 2007. Bacia do Araripe. Boletim de Geociências da Petrobras 15, 371- 389.

Assine, M. L., Perinotto, J. A. J., Neumann, V. H., Custódio, M. A., Varejão, F. G., Mescolotti, P. C., 2014. Sequências Deposicionais do Andar Alagoas (Aptiano superior) da Bacia do Araripe, Nordeste do Brasil. Boletim de Geociências da Petrobras, 22: 328.

Bobco, F.E.R., Goldberg, K., Bardola, T. P., 2017. Modelo deposicional do Membro Ipubi (Bacia do Araripe, nordeste do Brasil) a partir da caracterização faciológica, petrográfica e isotópica dos evaporitos. Pesquisas em Geociências, 44: 431-451.

Carvalho, I. S, Mendes, J. C., Costa, T., 2013. The role of fracturing and mineralogical alteration of basement gneiss in the oil exhsudation in the Sousa Basin (Lower Cretaceous), Northeastern Brazil. Journal of South American Earth Sciences, 47: 47-54.
Casilli, A., Silva, R. C., Laakia, J., Oliveira, C. J., Ferreira, A. A., Loureiro, M. R. B., Neto, F. R. A., 2014. High resolution molecular organic geochemistry assessment of Brazilian lacustrine crude oils. Organic geochemistry, 68: 61-70.

Castro, R.G., Lúcio, T., Fambrini, G.L., Souza Neto, J.A., Pereira, R., 2017. Caracterização Geoquímica dos Folhelhos Betuminosos da Formação Ipubi, Bacia do Araripe, NE do Brasil. Geochimica Brasiliensis, 31: 11-27.

Coimbra, J.C., Arai, M., Carreño, A.L., 2002. Biostratigraphy of Lower Cretaceous microfossils from the Araripe basin, northeastern Brazil. Geobios, 35: 687-698.

Barros Penteado, H. L., Behar, F., 2000. Geochemical Characterization and Compositional Evolution of the Gomo Member Source Rocks in the Reconcavo Basin, Brazil. AAPG Memoir 73, In: M.R. Mello, J.B. Katz, (Eds) Petroleum systems of South Atlantic margins: AAPG memoir, p. 179-194

Delgado, T.V.O., 2012. Maturação artificial de rochas geradoras de petróleo-carcaterização

geoquímicas e petrografia de folhelhos da Formação Ipubi, Grupo Santana, Bacia do Araripe, PE-CE. Trabalho de conclusão de curso, Graduação em GeologiaUFRGS, 79p.

Fabin, C.E., Correia Filho, O.J., Alencar, M.L., Barbosa, J.A., Mliranda, T.S.D., Neumann, V.H.L.M., Santana, F. R. D., 2018. Stratigraphic Relations of the Ipubi Formation: Siliciclastic-Evaporitic Succession of the Araripe Basin. Anais Da Academia Brasileira de Ciências, 90: 2049-2071.

Fambrini, G.L., Neumann, V.H.M.L., Barros, C.L., Galm, P.C., Agostinho, S.M., Araújo J.T., Menezes-Filho, J.A.B., 2012. 
Análise de fácies da Formação Brejo Santo, Bacia do Araripe, Nordeste do Brasil: implicações paleogeográficas. Estudos Geológicos, 22: 131-155.

Fambrini, G.L., Menezes-Filho, J.A., Jesuíno, P.C.L., Araújo, J.T., Durval, L.G.L., Neumann, V.H.L.M., 2015. Sucessão Faciológica da Formação Barbalha, Bacia do Araripe, Nordeste do Brasil. Estudos Geológicos, 25: 137-164.

Fambrini, G.L., Silva-Filho, W.F., Lemos, D.R., Silvestre, D.C., Araújo, J.T., Menezes-Filho, J.A.B., Tesser-Júnior, S., Neumann, $\quad$ V.H.L.M., 2019. Análise tectonossedimentar das fases início de rifte e clímax de rifte da Bacia do Araripe, Nordeste do Brasil. Geologia USP, 19: 205236.

Goldberg, K., Premaor, E., Bardola, T., Souza, P. A., 2019. Aptian marine ingression in the Araripe Basin: Implications for paleogeographic reconstruction and evaporite accumulation. Marine and Petroleum Geology, 107: 214-221.

Gonzalez, L.C.D., Mastalerz, M., Filho, J. G. M., 2020. Application of organic facies and biomarkers in characterization of paleoenvironmental conditions and maturity of sediments from the Codó Formation in the westcentral part of the São Luís Basin, Brazil. International Journal of Coal Geology, 103482.

Hockun, K., Mollenhauer, G., Ho, S.L., Hefter, J., Ohlendorf, C., Zolitschka, B., Mayr, C., Lucke, A., Schefu, E., 2016. Using distributions and stable isotopes of $\mathrm{n}$-alkanes to disentangle organic matter contributions to sediments of Laguna Potrok Aike, Argentina. Organic Geochemistry. 102: 110119.
Killops, S. D., Killops, V. J., 2005. Introduction to organic geochemistry. Blackweel, Oxford, 406p.

Lúcio, T., Souza Neto, J.A., Selby, D., 2020. Late Barremian / Early Aptian Re-Os age of the Ipubi Formation black shales: Stratigraphic and paleoenvironmental implications for Araripe Basin, northeastern Brazil. Journal of South American Earth Sciences, 102699.

Lúcio, T., Almeida, C.M.T., PachecoFilho, J.G.A., Araújo, J.C.M., Souza Neto, J.A., Pereira, R., 2016. Grau de maturação dos hidrocarbonetos dos folhelhos pirobetuminosos da Formação Ipubi, Bacia do Arraripe: Um estudo integrado de termogravimetria, cromatografia e espectroscopia na região do infravermelho. Estudos Geológicos, 26: 81-97.

Nascimento Jr, D.R., Silva Filho, W.F., Freire Jr, J.G., Santos, F.H., 2016. Syngenetic and Diagenetic Features of Evaporite-Lutite Sucessions of the Ipubi Formation, Araripe Basin, Santana do Cariri, NE Brazil. Journal of South America Earth Science, 72: 315327.

Neumann, V. H., Assine, M. L., 2015. Stratigraphic proposal to Post-Rift I Tectonic-Sedimentary Sequence of Araripe Basin, Northeastern Brazil. 2nd International Congress on Stratigraphy...,,Volume único p.1.

Neumann, $\quad$ V.H.M.L., 1999. Estratigrafía, Sedimentología, Geoquímica y Diagénesis de los Sistemas Lacustres AptiensesAlbienses de la Cuenca de Araripe (Noreste de Brasil). Tese de Doutorado, Pós graduação em Geoquímica e Estratigrafis-UB. 
Pereira, R., de Lima, F. J., Simbras, F. M., Bittar, S. M. B., Kellner, A. W. A., Saraiva, A. Á. F., Oliveira, G. R., 2019. Biomarker signatures of Cretaceous Gondwana amber from Ipubi Formation (Araripe Basin, Brazil) and their palaeobotanical significance. Journal of South American Earth Sciences, 102413.

Peters, K.E. Moldowan, J.M., 1993. The Biomarker Guide: Interpreting Molecular Fossils in Petroleum and Ancient Sediments. PrenticeHall Inc, New Jersey, 363p.

Peters, K.E. Walters, C.C., Moldowan, J.M., 2005. The Biomarker Guide: Biomarkers and Isotopes in the Environment and Human History. Cambridge, Cambridge University Press. 488p.

Silvestre, D.C., Fambrini, G.L., Santos, A.L.F., 2017. Caracterização Faciológica das formações Cariri e Brejo Santo em afloramentos a NE do município de Missão Velha (Ceará, Brasil). Estudos Geológicos, 27: 19-33

Souza Neto, J.A, Mort, H, Pereira, R, Barbosa, J.A, Neumann, V.H.L.M, Vortish, V, Correia Filho, O.J, Brandão, A.L.S., Pacheco, J.G. 2013. Carbonaceous Shales in the Araripe Basin, NE Brazil: A Potential Shale Gas Reservoir. In: AAPG Conference, 2013 ..., volume único p. 1-22
Souza, D.M., Piovesan, E.K., Neumann, V.H.L.M., 2017. Ostracodes do Aptiano-Albiano da Bacia do Araripe: Implicações Paleoambientais e Bioestratigráficas. Estudos Geológicos, 27: 3-18.

Scherer, C.M.S., Jardim de Sá, E. F., Córdoba, V. C., Sousa, D. do C., Aquino, M. M., Canelas Cardoso, F. M., 2014. Tectono-stratigraphic evolution of the Upper JurassicNeocomian rift succession, Araripe Basin, Northeast Brazil. Journal of South American Earth Sciences, 49: 106-122.

Tissot, B.P., Welte, D.H., 1984. Petroleum Formation and Occurrence. Springer-Verlag, Berlin, 699p.

Valença, L.M.M., Neumann, V.H.L.M., Mabesoone, J.M., 2003. An overview on CallovianCenomanian intracratonic basins of Northeast Brazil: Onshore stratigraphic record of the opening of the Southern Atlantic. Geologica Acta, 1: 261-275

Xia, L., Cao, J., Hu, S., Li, S., Shi, C., 2019. Organic geochemistry, petrology, and conventional and unconventional hydrocarbon resource potential of Paleogene saline source rocks in eastern China: The Biyang Sag of the Nanxiang Basin. Marine and Petroleum Geology, 101: 343-354. 